\title{
Sodium Lactate, D-
}

National Cancer Institute

\section{Source}

National Cancer Institute. Sodium Lactate, D-. NCI Thesaurus. Code C61718.

The sodium salt of the dextro isomer of lactic acid with alkalinizing and electrolyte replenishing property. Upon metabolism, sodium lactate D is converted to bicarbonate, thereby increasing plasma bicarbonate, which facilitates removal of hydrogen ion and lactate from blood stream and leads to raised blood pH. 